\title{
CONTRIBUTION A L'ÉtUdE DES MÉthodes DE RECHERCHE DES NEUTRALISANTS DANS LES POUDRES DE LAIT ÉCRÉMÉ
}

\author{
par
}

L. SERRES, S. AMARIGLIO, L. BENARD, D. ROBINET

Laboratoire Central du Service Technique Interprofessionnel du Lait

Les méthodes de recherche des neutralisants ajoutés au lait sont nombreuses. En effet, les substances dosées peuvent être différentes et chaque dosage peut être effectué selon des techniques variées.

Dans le but de faire un choix parmi les méthodes mises à la disposition des analystes, nos travaux ont été conduits selon les plan suivant :

I. - Revue des diverses méthodes.

1) Dosage de l'alcalinité des cendres.

2) Dosage du sodium, du potassium, du calcium et de l'ammoniaque.

3) Dosage des lactates et de l'acide lactique.

II. - Essais expérimentaux.

1) Etude comparative de quelques méthodes de recherche des neutralisants dans les laits liquides :

- alcalinité des cendres : méthode de l'A. O. A. C. (1).

méthode des « Netherlands Regulations ».

- sodium, potassium,

calcium : méthode par photométrie de flamme.

- ammoniaque : méthode chimique.

- lactates et acide lactique : méthode de Davidson.

: méthode de Venekamp et Kruisheer.

2) Etude comparée des résultats de ces méthodes appliquées aux laits liquides et aux poudres correspondantes.

III. - Application à l'analyse d'échantillons de laits en poudre de provenances diverses.

\section{I. - REVUE DES DIVERSES MÉTHODES}

\section{Dosage de l'alcalinité des cendres.}

\section{a) Principe}

On peut exprimer, de manière globale, la teneur du lait en substances minérales par la teneur en cendres déterminée après incinération à température relativement basse $\left(550^{\circ}\right.$ C. $)$. Mais le taux des cendres varie trop fortement, suivant l'origine du lait, pour que l'on puisse l'utiliser pour la recherche des neutralisants fixes.

(1) Association of Official Agricultural Chemists (U. S. A.). 
L'alcalinité des cendres, par contre, varie dans des limites plus étroites. C'est pourquoi ce dosage est fréquemment utilisé dans ce but. L'alcalinité des cendres provenant de lait neutralisé par des produits basiques s'élève au-dessus des valeurs moyennes.

\section{b) Méthodes}

Il existe plusieurs méthodes; certaines présentent des inconvénients :

1. Le titrage est fait sur la solution aqueuse et filtrée des cendres; par exemple, selon la méthode indiquée par Thieulin et VuILLAUMe [1]. Toutefois, il existe deux causes d'erreur :

a) une partie de l'agent alcalin reste dans la fraction insoluble sous forme de phosphates complexes du type $\mathrm{CaNaPO}_{4}$ et aussi, à l'état de traces, de carbonates doubles du type $\mathrm{CaNa}_{2}\left(\mathrm{CO}_{3}\right)_{3}$, (WICHMANN [2]).

b) Les phosphates solubles interfèrent dans le dosage alcalimétrique.

2. La méthode simple de titrage alcalimétrique a été améliorée pour éviter l'interférence de phosphates; on utilise actuellement deux sortes de méthodes, le titrage étant fait sur la solution des cendres dans un acide titré :

- Le titrage est fait en deux temps; en premier lieu, on titre au point de virage du jaune de diméthyle (ou jaune de beurre) environ à $p \mathrm{H} 4$; en second lieu, on titre au point de virage de la phénol phtaléine (environ $p \mathbf{H} 8,5$ ) en présence d'oxalate de potassium. Avec les deux valeurs obtenues on calcule différents indices. C'est la méthode de FARnsteiner [3] modifiée par Kolthoff [4] et adoptée par les "Netherlands Regulations " [4]. Brouwer [5] a donné une explication de ces indices. L'un d'eux correspond à la neutralisation des carbonates et des oxydes, les phosphates étant exclus;

- Le titrage est fait en présence de chlorure de calcium ; c'est le principe de la méthode mise au point par HILLIG [6] et adoptée par l'A. O. A. C. [7]. En suivant cette technique, on dose également une partie des phosphates naturels du lait.

\section{c) Résultats}

Kruisheer, Van der Burg, Krol, Tiersma, Venekamp [8] rappellent l'importance des variations naturelles de l'indice d'alcalinité :

\section{8,0 à 23,4 selon VAN GuLIK,}

17,55 à 22,85 selon VAN DER BURG.

Lorsqu'on ne connaît pas l'alcalinité des cendres du lait d'origine, il est difficile dans de nombreux cas de conclure que le lait 
a été neutralisé, étant donné l'amplitude des variations naturelles [8] [9].

Ces méthodes sont insuffisantes pour déterminer de faibles neutralisations.

\section{Dosage du sodium, du potassium, du calcium et de l'am- moniaque.}

a) Principe

Il existe dans le lait des rapports de valeur constante : travaux de W. L. Davies [10], confirmés par ceux de Honwitz [11], qui indiquent les rapports moyens de 0,47 pour $\mathrm{Na} / \mathrm{Cl}$ et 1,3 pour $\mathrm{P} / \mathrm{Ca}$.

\section{b) Méthodes}

๔) Méthodes chimiques.

- Pour le dosage du sodium, Sato et Murata [12] emploient le réactif au pyroantimoniate. La méthode à l'acétate d'uranyle et de magnésium décrite par KAHANE [13] a été appliquée au lait par Treille [14].

- Le dosage du potassium a été étudié par VLadesco [15], par SAto et Murata [12]. Ils emploient le réactif au cobaltinitrite de soude.

- Les études sur le dosage du calcium sont relativement nombreuses en raison du rôle que joue cet élément en technique laitière et dans l'alimentation humaine. Le dosage par précipitation à l'état d'oxalate a été longtemps employé. Citons les travaux de SATo et Murata [16], de Burr et Witt [17], de Vladesco [18], de Schwarz et MU MM [19]. La méthode aux complexones est actuellement en faveur (Jenness [20]).

- Pour le dosage de l'ammonium, les méthodes par distillation après digestion sont classiques. Celle de PARNAS a été utilisée par Niemczycki et Gerhardt [21], par Heller et Swiechowska [22]. Le principe est le suivant : le borate de soude empêche la décomposition des amines ou des amino-acides et déplace l'ammoniac qui, entraîné par la vapeur d'eau, est reçu dans une solution d'acide titré. Perkins [23] distille le filtrat alcoolique du lait en présence d'oxyde de magnésium.

- Afin de calculer le rapport $\mathrm{Na} / \mathrm{Cl}$, le dosage des chlorures peut être effectué par une méthode dérivée de celle de Schalms et Schales [24] ou par la méthode officielle de CHarpentierVOLHARD.

ß) Méthode physique.

La photométrie de flamme a été appliquée au dosage des métaux dans le lait depuis 1939 par ZIEGLER [25] qui dosait uni- 
quement le potassium; puis par KRIENKE et GAMmon [26] qui dosent à la fois $\mathrm{K}, \mathrm{Na}$ et $\mathrm{Ca}$. De nombreux auteurs l'ont utilisée par la suite [27] [28]...

La solution à analyser est pulvérisée de façon continue dans la flamme du photomètre; les atomes métalliques émettent un rayonnement de longueur d'onde spécifique ; celui-ci, sélectionné par un filtre, est transformé par une cellule photoélectrique; le courant produit est mesuré par un galvanomètre. Les divers types d'appareils ont des possibilités très différentes. Le calcium, en présence de phosphates, ne peut être dosé qu'avec des appareils perfectionnés. Il faut, d'autre part, tenir compte des interférences des cations entre eux; celles-ci varient avec le type d'appareil utilisé.

La préparation de la solution diffère suivant les auteurs :

- dilution directe à l'eau bidistillée : CoRMreR [29], VAN DER Have et Mulder [30];

- défécation par l'acide trichloracétique : ALArs [31];

- séparation des cations sur colonnes échangeuses d'ions: WenNer [32];

- minéralisation par les acides perchlorique et nitrique.

c) Résultats

La teneur du lait normal en $\mathrm{K}, \mathrm{Na}, \mathrm{Ca}, \mathrm{NH}_{4}$ dépend du stade de lactation [33], de la race [34] et varie suivant l'alimentation [35] [36] et les saisons.

- Le taux de sodium diminue en hiver (BLANCHETIERE [37]) est élevé dans le colostrum (Garketr et Overman [38]) ; NottBoHm cité par KRUISHeER [8] indique les résultats extrêmes de 4,09 et 5,92 milligrammes $\mathrm{Na}$ par gramme d'extrait sec dégraissé. TreILLE a obtenu 0,28 à 0,41 gramme de Na par litre de lait normal.

- Le taux de potassium diminue également en hiver (BLANCHETIERE [37]); il est plutôt faible dans le colostrum (GARRETT et Overman [38]).

- Le taux de calcium varie peu au cours de l'année. D'après Blanchetiere [37] et Kulagina [39], ce taux augmente en hiver ; d'après VANSchou BROEK [40], il diminue légèrement en été. AuRIOL et Mocquot [41] montrent que dans la région du Jura, la teneur en calcium va en augmentant légèrement de mars à septembre (vie au pâturage) et diminue progressivement d'octobre à février (vie à l'étable). Rombauts et col. [33] mettent en évidence l'influence de la race; le lait des vaches normandes est, en moyenne, plus riche en calcium que celui des vaches de races pie-rouge et française frisonne pie-noire. RICHMOND [42] indique comme amplitude normale des variations 1,25 à $1,36 \%$. 


\section{Dosage des lactates et de l'acide lactique.}

\section{a) Principe}

La désacidification du lait est en fait la neutralisation des acides organiques, principalement de l'acide lactique produit par fermentation après la traite. Pour mettre en évidence l'addition de neutralisant, il suffit de doser les lactates formés. Or les procédés de dosage connus ne permettent pas de différencier les lactates de l'acide lactique.

Le lait frais a une teneur apparente en acide lactique très faible; Davidson [43] l'évalue à $15-20 \mathrm{mg}$./1 et Ling [44] à $30 \mathrm{mg}$./1.

Lorsque la tereur en acide lactique est faible et l'acidité titrable normale, on peut admettre que l'échantillon n'a pas été désacidifié. Lorsque la teneur est élevée, le lait ayant subi une fermentation lactique importante, l'acidité titrable doit s'élever dans les mêmes proportions. Si elle n'augmente pas, le degré théorique de neutralisation apparaît par comparaison avec l'acidité du lait frais. Un autre moyen consiste à déterminer le $p \mathbf{H}$ et à le comparer au $p \mathbf{H}$ normal du lait frais lorsque ce dernier est connu [45].

\section{b) Méthodes}

๙) Méthodes colorimétriques.

Le dosage nécessite la séparation préalable des protéines ou des protéines et du lactose; cette séparation peut être effectuée selon plusieurs techniques :

- défécation : chlorure de baryum, soude et sulfate de zinc [8]; sulfate de cuivre et chaux [46]; oxyde de fer dialysé [45];

- passage sur colonnes d'échangeurs d'ions : résine [47], [48], [49] ou acide silicique [50]. Ces techniques de purification donnent de bons résultats, mais elles sont longues. Elles peuvent être utilisées pour l'identification des acides organiques présents dans le lait.

Plusieurs procédés ont été décrits pour le dosage colorimétrique ; ils reposent sur deux réactions :

- oxydation par l'acide sulfurique concentré en acétaldéhyde et mise en cuvre d'une des réactions colorées de ce corps : réaction de MeNDel et GoLdSCHEIDER avec le vératrol appliquée au lait par HeInemann [51] et PAULSen [52]; réaction de Eegriwe avec le para-hydroxydiphényl utilisée par Davinson [43]; KLeinert [53] a fait une critique de cette dernière technique;

- réaction de l'acide lactique avec le chlorure ferrique, selon LANDWEHR. Elle a été appliquée au lait par HILLIG et adoptée par l'A. O. A. C. [54]. Elle a été également utilisée par LING [44] et par Venekamp et KrUisheer [45] qui ont mis au point une méthode rapide de routine. Cette réaction est en réalité une réaction 
des groupements hydroxyl; elle manque de spécificité ; toutefois, dans le lait, les interférences du lactose et de l'acide citrique paraissent peu gênantes [45].

ß) Méthode potentiométrique.

WoIdich et SCHMID [55] mesurent le nombre de millilitres de $\mathrm{HCl} 0,25 \mathrm{~N}$ nécessaire pour porter 25 millilitres de lait à un $p \mathrm{H}$ de 2,7 : le pouvoir tampon dans la zone acide des $p H$ dépend notamment de la quantité d'acide lactique.

\section{c) Résultats}

Leur interprétation est délicate car elle fait intervenir l'acidité naturelle du lait frais dont les variations sont bien connues [8], ce qui diminue la précision des résultats.

\section{II. - ESSAIS EXPERIMENTAUX}

\section{1. Étude comparative de quelques méthodes de recherche des neutralisants dans les laits liquides.}

Pour déterminer la valeur des trois groupes de méthodes, nous faisons l'étude critique de chacune d'elles sur les points suivants : interprétation des résultats, reproductibilité, facilité d'exécution pratique.

L'analyse a été faite sur un même lait successivement :

- à l'état frais,

- acidifié,

- neutralisé.

Il était nécessaire de disposer d'échantillons témoins de lait frais sans acidité acquise et sans neutralisant. Ils ont été prélevés aseptiquement au moment de la traite, refroidis, transportés rapidement au laboratoire, écrémés à $25-30^{\circ} \mathrm{C}$., refroidis à nouveau et analysés aussitôt. Toutes ces opérations ont été réalisées dans une seule matinée.

Ensuite, ils ont été acidifiés par ensemencement de ferments lactiques à la dose de $0,5 \%$ et incubation à $30^{\circ} \mathrm{C}$. Leur acidité a été limitée à un maximum de $0,3 \%$ acide lactique (30 Dornic).

Enfin, chaque échantillon a été divisé en plusieurs lots neutralisés respectivement par l'un des produits suivants : bicarbonate de sodium, carbonate de sodium, soude, chaux, ammoniaque.

1) Dosage de l'alcalinité des cendres.

- Méthode de l'A. O. A. C. [7] ;

- Méthode hollandaise [4].

Les essais effectués sur 15 échantillons de lait frais de la région parisienne montrent l'amplitude des variations naturelles : 200 à 
550 milligrammes en $\mathrm{Na}_{2} \mathrm{CO}_{3}$ (méthode A. O. A. C.), indice 20,5 à 23,5 (méthode hollandaise).

Sur des laits de mélange d'autres régions, l'amplitude est respectivement de 300 à 500 milligrammes et de 21 à 23,25 (exemples dans le tableau V).

Sur les laits acides (sans neutralisation), par la méthode de l'A. O. A. C., l'alcalinité est sensiblement la même que pour les laits frais; par la méthode hollandaise, l'indice est parfois plus élevé.

Pour le lait désacidifié par les composés de $\mathrm{Na}$ et de $\mathrm{Ca}$, on retrouve les quantités de neutralisant ajoutées en utilisant la méthode de l'A. O. A. C. Par la méthode hollandaise, on constate également une augmentation de l'indice en corrélation avec les doses ajoutées. Mais la détermination de l'alcalinité des cendres est évidemment en défaut dans le cas des laits désacidifiés par l'ammoniaque; elle indique même parfois une légère diminution,

Ces méthodes sont done sensibles à l'addition de composés alcalins et alcalino-terreux. Mais, pour calculer la quantité ajoutée, il est nécessaire de connaître la composition initiale du lait frais. Cette condition n'est pratiquement jamais remplie.

D'une façon générale et en reprenant les seuils trouvés (300 à 500 milligrammes), pour que le chimiste ait la certitude de l'addition d'un produit alcalin, un lait de grand mélange et de faible alcalinité naturelle devrait être neutralisé avec plus de $200 \mathrm{mg} . / 1$. de $\mathrm{Na}_{2} \mathrm{CO}_{3}$ (ou son équivalent), ce qui correspond à 4 décigrammes d'acide lactique par litre (40 Dornic).

La méthode de l'A. O. A. C. est reproductible, la méthode hollandaise l'est moins, notamment à cause du manque de netteté des virages.

Il est nécessaire d'attirer l'attention sur certaines conditions opératoires :

Méthode A.O.A.C.: réglage exact de la température du four, entraînement intégral des cendres qui restent parfois collées sur la paroi de la capsule, absence de projections au cours de l'ébullition.

Méthode hollandaise: chauffage modéré indispensable.

A condition de prendre toutes les précautions énumérées cidessus, ces méthodes peuvent être utilisées pour les analyses en série. Notons qu'un opérateur peut, en une journée, exécuter une série de dix analyses, le lait ayant été préalablement desséché.

2) Dosage du sodium, du potassium, du calcium et de l'ammoniaque.

a) Le dosage des alcalins et alcalino-terreux a été effectué par photométrie de flamme. Nous avons utilisé l'appareil d'EPPEN- 
DORF (1) qui offre des possibilités nouvelles pour les dosages des métaux alcalins et alcalino-terreux et qui présente une sélectivité poussée. GuEguen et Rombauts [57] ont montré les possibilités de dosage dans le cas du lait.

Sur 25 échantillons de laits frais individuels de la région parisienne, les teneurs par litre de lait varient ainsi :

300 à $500 \mathrm{mg}$. de $\mathrm{Na}$,

1.500 à $2.000 \mathrm{mg}$. de $\mathrm{K}$,

1.000 à $1.450 \mathrm{mg}$. de Ca.

Sur 10 laits de mélange de provenance diverses, nous obtenons :

375 à $475 \mathrm{mg}$. de $\mathrm{Na}$,

1.500 à $1.750 \mathrm{mg}$. de $\mathrm{K}$,

1.100 à $1.250 \mathrm{mg}$. de Ca (exemples dans le tableau VI).

L'acidification du lait n'apporte pas de modification au résultat de ces dosages.

Sur lait désacidifié par les composés de $\mathrm{Na}$ et $\mathrm{Ca}$, on retrouve les quantités ajoutées, mais pour calculer ces dernières, il est utile, comme dans les précédents dosages, de connaître la composition initiale du lait. Dans un lait de mélänge à faible teneur initiale, on ne peut être certain de l'addition de neutralisant que si la dose est importante (100 mg. de Na par litre correspondent à 4 dg. d'acide lactique ou $4^{\circ}$ Dornic).

Cette méthode donne des résultats reproductibles; cependant, pour le calcium, il est nécessaire d'étalonner fréquemment l'appareil.

Du point de vue pratique, le dosage est simple et rapide; un opérateur peut, en une journée, faire les trois déterminations $\mathrm{Na}$, $\mathrm{K}$ et Ca sur 40 échantillons de lait liquide (ou sur 20 à 25 de lait sec).

Simultanément, des analyses ont été effectuées pour vérifier la constance du rapport $\mathrm{Na} / \mathrm{Cl}$.

La méthode dérivée de celle de Schales et Schales et la méthode de CHARPEnTIER-VolHaRD donnent des résultats comparables pour le dosage des chlorures. Le taux est généralement voisin de 1,7 g./l. dans les laits de mélange et le calcul donne un rapport $\mathrm{Na} / \mathrm{Cl}$ de 0,45 à 0,50 .

b) Le dosage de l'ạmmoniaque a été réalisé par une méthode chimique : défécation puis entraînement de $\mathrm{NH}_{3}$ par l'alcool éthylique (voir méthode en annexe).

Sur les laits frais, la teneur est très faible, toujours inférieure à $6 \mathrm{mg} \cdot / 1$.

(1) Nous remercions la Station de Biochimie du Centre National de Recherches Zootechniques de Jouy-en-Josas, dont le Directeur, M. François, a mis à notre disposition l'appareil Eppendorf (Netheler et Hinz, fabricants). 
Sur les laits acidifiés par les ferments lactiques, la teneur reste inchangée.

Sur les laits désacidifiés par l'ammoniaque, on retrouve approximativement la dose ajoutée.

Cette méthode est reproductible; elle est d'exécution facile et assez rapide. Un opérateur peut faire dix analyses en série par jour.

3) Dosage des lactates et de l'acide lactique. - Comparaison avec l'acidité titrable.

Comme il a été indiqué (première partie, $3^{\mathrm{e}} a$ ), pour déceler la neutralisation, le dosage proprement dit doit s'accompagner de la détermination de l'acidité ionique ou de l'acidité titrable (1). Bien que celle-ci présente certains inconvénients, elle a été préférée en raison de sa simplicité.

Pour le dosage de l'acide lactique libre et combiné (acide lactique + lactates), nous avons étudié :

- la méthode de Davidson,

- la méthode de Venekamp et Kruisheer.

\section{a) La méthode de Davidson}

C'est une méthode précise. Des quantités déterminées de lactate de lithium ajoutées au lait sont retrouvées avec une approximation de $\pm 5 \%$ pour les quantités inférieures à $100 \mathrm{mg} . / \mathrm{l}$, et de $\pm 3 \%$ pour les quantités de 100 à $1.000 \mathrm{mg}$./l. Mais son exécution doit être minutieuse ; elle est relativement longue et nécessite un appareillage assez important. DAVIDSON [43] et KLEINERT [53] ont indiqué les précautions indispensables. Soulignons que l'acide sulfurique doit être absolument pur; pour le nettoyage de la verrerie, les détergents organiques sont à proscrire.

Nous pensons qu'elle ne convient pas aux analyses en série. Elle peut servir de méthode de référence lorsque la méthode rapide de Venekamp et Kr uisheer donne des résultats douteux.

Les résultats suivants ont été obtenus sur les échantillons de lait frais (voir tableau I).

Nous trouvons pour les laits frais des teneurs en acide dosable variant de 0,16 à $0,45 \mathrm{dg} . / 1$. ; ce sont des valeurs normales (voir plus haut). L'acidité naturelle est très proche de l'acidité titrable. L'amplitude des variations, d'après le tableau I, est de $3^{\circ}$ Dornic.

Dans les laits acides, nous constatons une corrélation entre l'augmentation d'acidité de titration et l'augmentation d'acide lactique dosé (voir tableau II).

(1) Journal officiel du 23 avril 1954. 


\section{TABLEAU I}

\section{AGIDITE DES LAITS FRAIS INDIVIDUELS}

(en degrés Dornic ou dg/1)

\begin{tabular}{|c|c|c|c|}
\hline Origine & Acidité titrable & $\begin{array}{l}\text { Acidité correspon- } \\
\text { dant à l'acide } \\
\text { lactique dose } \\
\text { (Davidson) }\end{array}$ & Acidité naturelle \\
\hline & At & Al & $\mathrm{An}=\mathrm{At}-\mathrm{Al}$ \\
\hline 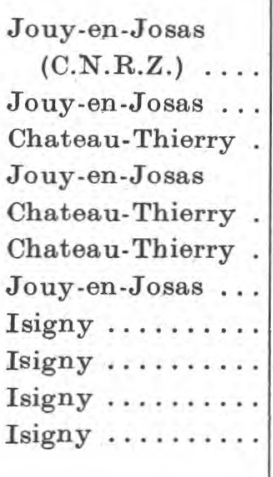 & $\begin{array}{l}14,5 \\
14 \\
15,5 \\
14 \\
13,5 \\
12,5 \\
13 \\
14 \\
14 \\
13,5 \\
13,5\end{array}$ & $\begin{array}{l}0,16 \\
0,16 \\
0,175 \\
0,19 \\
0,19 \\
0,22 \\
0,25 \\
0,42 \\
0,40 \\
0,45 \\
0,42\end{array}$ & \begin{tabular}{|r|}
14,34 \\
13,84 \\
15,33 \\
13,81 \\
13,31 \\
12,28 \\
12,75 \\
13,52 \\
13,60 \\
13,05 \\
13,08 \\
\end{tabular} \\
\hline
\end{tabular}

L'accroissement d'acidité est le même à $1^{0}$ Dornic près. Bien que la mesure de l'acidité titrable ne soit précise qu'à $\pm 0^{\circ} 5$ Dornic, on peut conclure que l'augmentation d'acide lactique dosé par voie chimique et l'augmentation d'acidité titrable sont concordantes.

Examinons, à présent, la répercussion sur le résultat fourni par les deux méthodes, de la neutralisation par différents désacidifiants (voir tableau III).

Le dosage des lactates et de l'acide lactique donne, comme il fallait s'y attendre, le même résultat sur les laits acides et sur les mêmes laits neutralisés. Lorsqu'on neutralise par la soude et l'ammoniaque, la baisse d'acidité titrable obtenue correspond bien à la baisse prévue. Avec l'emploi de carbonate ou de bicarbonate la baisse d'acidité est plus faible; en effet, le gaz carbonique issu de la réaction se dissout en partie dans le lait; il suffit de porter ce lait à l'ébullition afin de chasser le gaz carbonique pour que la baisse d'acidité obtenue corresponde à celle prévue. 
TABLEAU II

GOMPARAISON DES MESURES DE L'AGCROISSEMENT D'AGIDITE

(en degrés Dornic ou dg/1)

\begin{tabular}{|c|c|c|c|c|c|c|}
\hline \multicolumn{2}{|c|}{ Avant incubation } & \multicolumn{2}{|c|}{ Après incubation } & \multicolumn{3}{|c|}{ Accroissement } \\
\hline Acidité titrable & $\begin{array}{c}\text { Acide lactique } \\
\text { dosé (Davidson) }\end{array}$ & Acidité titrable & $\begin{array}{c}\text { Acide lactique } \\
\text { dosé (Davidson) }\end{array}$ & Acidité titrable & $\begin{array}{c}\text { Acide lactique } \\
\text { dosé (Davidson) }\end{array}$ & Différence \\
\hline 14,5 & 0,5 & 16,5 & 2,3 & 2 & 1,8 & $+0,2$ \\
\hline 15 & 1 & 17,5 & 3,5 & 2,5 & 2,5 & 0 \\
\hline 16 & 2,45 & 19,5 & 5,5 & 3,5 & 3,05 & $+0,45$ \\
\hline 15 & 1,5 & 18,5 & 6 & 3,5 & 4,5 & -1 \\
\hline 15,5 & 1,5 & 21 & 7,5 & 5,5 & 6,0 & $-0,5$ \\
\hline 16,5 & 1,5 & 24 & 8,7 & 7,5 & 7,2 & $+0,3$ \\
\hline 15,5 & 1,6 & 26 & 10,5 & 10,5 & 9,0 & $+1,5$ \\
\hline 15,5 & 1,5 & 25 & 11 & 9,5 & 9,5 & 0 \\
\hline 15 & 1,0 & 25 & 10,7 & 10 & 9,7 & $+0,3$ \\
\hline 15,5 & 1,5 & 27 & 12,5 & 11,5 & 11,0 & $+0,5$ \\
\hline 17,5 & 2,5 & 33,5 & 17,5 & 16 & 15,0 & +1 \\
\hline 17 & 3,8 & 37 & 24 & 20 & 20,2 & $-0,2$ \\
\hline
\end{tabular}


TABLEAU III

INFLUENGE DE LA NEUTRALISATION SUR LE DOSAGE DES LAGTATES ET SUR LA MESURE DE L'ACIDITÉ DORNIG (en degrés Dornic og dù/1)

\begin{tabular}{|c|c|c|c|c|c|c|c|}
\hline \multirow[b]{2}{*}{$\begin{array}{c}\text { Neutralisant } \\
\text { employé }\end{array}$} & \multirow[b]{2}{*}{$\begin{array}{c}\text { Baisse } \\
\text { d'acidité } \\
\text { prévue }\end{array}$} & \multicolumn{3}{|c|}{ Acide lactique dosé (Méthode Davidson) } & \multicolumn{3}{|c|}{ Acidité titrable (Méthode Dornic) } \\
\hline & & $\begin{array}{l}\text { lait } \\
\text { acide }\end{array}$ & $\begin{array}{c}\text { lait } \\
\text { neutralisé }\end{array}$ & différence & $\begin{array}{r}\text { lait } \\
\text { acide }\end{array}$ & $\begin{array}{c}\text { lait } \\
\text { neutralisé }\end{array}$ & $\begin{array}{l}\text { baisse d'aci- } \\
\text { dité obtenue }\end{array}$ \\
\hline $\mathrm{NaOH} \ldots$. & 5 & 6,05 & 6,05 & 0,0 & 21,5 & 17 & 4,5 \\
\hline $\mathrm{NaOH} \ldots$. & 5 & 6,25 & 6,25 & 0,0 & 21 & 16 & 5 \\
\hline $\mathrm{NH}_{4} \mathrm{OH} \ldots$ & 8 & 6,05 & 6,05 & 0,0 & 21,5 & 13,5 & 8 \\
\hline $\mathrm{Na}_{2} \mathrm{CO}_{3} \quad \ldots$ & 5 & 6,25 & 6,15 & 0,10 & 21 & 18 & 3 \\
\hline $\mathrm{HNaCO}_{3} \ldots$ & 6 & 6,05 & 5,95 & 0,10 & 21,5 & 20 & 1,5 \\
\hline $\mathrm{HNaCO}_{3} \ldots$ & 5 & 6,25 & 5,95 & 0,3 & 21 & 19 & 2 \\
\hline $\begin{array}{r}\mathrm{HNaCO}_{3} \text { et } \\
\text { ébullition. }\end{array}$ & 5 & 6,05 & 6,05 & 0,0 & 21,5 & 17 & 4,5 \\
\hline
\end{tabular}

TABLEAU IV

Gomparaison des Résultats obTENUS PAR LES DEUX MÉthodes

\begin{tabular}{|c|c|c|c|c|}
\hline \multirow{2}{*}{$\begin{array}{c}\text { Nature et origine } \\
\text { des laits }\end{array}$} & \multicolumn{4}{|c|}{ Acide lactique dosé exprimé en dg/l } \\
\hline & Davidson & $\begin{array}{l}\text { Venekamp et } \\
\text { Kruisheer }\end{array}$ & Différence & \\
\hline Frais : & & sing & & \\
\hline Jouy-en-Josas ..... & 0,16 & 0,50 & $+0,34$ & \\
\hline Chateau-Thierry ... & 0,175 & 0,30 & $+0,125$ & \\
\hline Chateau-Thierry ... & 0,19 & 0,20 & $+0,01$ & $\therefore$ \\
\hline Chateau-Thierry ... & 0,22 & 0,20 & $-0,02$ & \\
\hline Chateau-Thierry ... & 0,50 & 0,50 & 0,00 & \\
\hline Isigny $\ldots \ldots \ldots \ldots$ & 0,50 & 0,60 & $+0,10$ & \\
\hline Jouy-en-Josas $\ldots \ldots$ & 0,75 & 0,80 & $+0,05$ & \\
\hline Légèrement acides: & & & & \\
\hline Chateau-Thierry ... & 2,00 & 1,50 & $-0,50$ & \\
\hline Jouy-en-Josas .... & 2,10 & 2,30 & $+0,20$ & \\
\hline Chateau-Thierry ... & $2,4,0$ & 1,90 & $-0,50$ & \\
\hline Jouy-en-Josas .... & 3,30 & 3,20 & $-0,10$ & \\
\hline$\therefore$ & & 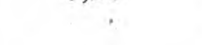 & & \\
\hline Acides : & & & & \\
\hline Isigny $\ldots \ldots \ldots \ldots$ & 7,00 & 7,90 & $+0,90$ & \\
\hline Isigny $\ldots \ldots \ldots \ldots$ & 7,40 & 8,10 & $+0,70$ & \\
\hline Isigny $\ldots \ldots \ldots \ldots$ & 7,75 & 8,00 & $+0,25$ & \\
\hline Jouy-en-Josas $\ldots \ldots$ & 8,20 & 7,50 & $-0,70$ & \\
\hline Isigny $\ldots \ldots \ldots \ldots$ & 8,40 & 8,00 & $-0,40$ & \\
\hline Jouy-en-Josas .... & 9,25 & 9,10 & $-0,15$ & \\
\hline Jouy-en-Josas .... & 9,85 & 9,80 & $-0,05$ & \\
\hline
\end{tabular}




\section{b) Méthode Venekamp et Kruisheer. - Comparaison avec la méthode Davidson}

La valeur de la méthode Venekamp et Kruisheer a été étudiée par comparaison avec la méthode Davidson pour un échantillonnage de laits frais et acides (tableau IV) et neutralisés (tableau VII).

Les résultats des analyses de laits frais et acides sont rassemblés dans le tableau $1 \mathrm{~V}$.

Dans ces échantillons non neutralisés, la différence est inférieure à $1 \mathrm{dg} . / 1$. Le rapport $\mathrm{x} / \sigma$ de la moyenne des écarts $(\mathrm{x}=0,0141)$ à l'écart type $(\sigma=0,0298)$ est faible et les deux séries de résultats sont comparables.

Si l'on tient compte du tableau VII concernant l'analyse des laits traités à l'usine, on obtient les valeurs $\mathrm{x}=0,394$ et $\sigma=0,119$. Le rapport $\mathrm{x} / \sigma$ étant supérieur à 2 , l'écart est significatif et les deux séries de résultats ne sont pas tout à fait comparables.

La corrélation entre les deux méthodes est done plus étroite dans les laits frais ou acides que dans les laits neutralisés. Dans ceuxci, les différences atteignent parfois 2 décigrammes d'acide lactique par litre ou $2^{\circ}$ Dornic (cas d'une neutralisation par la chaux, peu soluble).

La méthode de VENEKAMP et KR UISHEER présente l'avantage d'être plus simple que celle de Davidson. Elle ne nécessite qu'un appareillage courant ; elle est utilisable dans les laboratoires industriels possédant un photocolorimètre (1). Les seules précautions à prendre sont de se procurer $d u$ fer dialysé stable et de vérifier la courbe d'étalonnage à chaque changement de préparation du réactif.

La précision des dosages est suffisante. Lorsqu'on ajoute une quantité déterminée de lactate de lithium à un lait frais, l'analyse la révèle avec une approximation de $\pm 5 \%$. Cependant la réaction de coloration est spécifique du groupement $\mathrm{OH}$ et non de l'acide lactique [45]. Dans la pratique de l'analyse des laits, cela explique en partie les différences figurant dans les tableaux IV et VII qui, nous l'avons constaté, ne dépassent pas. $1^{\circ}$ Dornic dans les laits frais ou acides et $2^{\circ}$ Dornic dans les laits neutralisés.

Cette méthode est reproductible et convient particulièrement bien aux analyses en série ; un opérateur peut faire environ 70 déterminations par jour.

La méthode Dornic pour le dosage de l'acidité de titration est très rapide. Cependant, sa précision ne dépasse pas $0 \circ 5$ Dornic. Signalons qu'il ne faut en aucun cas ajouter de l'eau distillée au

(1) Les auteurs signalent l'utilisation possible, à défaut de photocolorimètre, d'une gamme colorimétrique standard. 
cours de la détermination car cela provoquerait un changement du degré d'ionisation du lait. D'autre part, il faut toujours ajouter la même quantité de la solution d'indicateur; la concentration de celle-ci en phénolphtaléine ne doit pas varier.

\section{c) Commentaire}

On sait que, pour un lait frais, l'acidité titrable représente l'acidité originelle du lait correspondant à la caséine, aux matières minérales acides et aux traces d'acides organiques, plus la quantité de solution alcaline consommée par les réactions secondaires dues aux phosphates [56].

Nous avons appelé, dans cet exposé "acidité naturelle » l'acidité de titration du lait à la traite diminuée de l'acidité correspondant aux traces d'acide lactique dosables, toujours présentes dans le lait frais (tableau I).

Dans un lait acidifié non neutralisé on a donc : acidité titrable $=$ acidité naturelle + acidité développée (équat. I).

Si le lait acidifié a été neutralisé, cette équation n'est plus vérifiée : l'acidité titrable est inférieure à la somme de l'acidité naturelle plus l'acidité développée, cette dernière étant déterminée par le dosage chimique de l'acide lactique.

Il faut souligner le fait qu'en pratique un terme de cette équation n'est jamais connu avec certitude : l'acidité naturelle. Or, il s'agit d'une valeur qui peut varier assez fortement dans les laits individuels; nous avons trouvé (tableau I) une moyenne de $13^{\circ} 5$ Dornic avec des variations limites de \pm 105 Dornic.

L'interprétation des résultats fournis par les méthodes de dosage de l'acide lactique est donc quelque peu limitée par l'existence de ce terme imprécis. Cependant, ces méthodes présentent l'avantage de mettre en évidence tous les neutralisants, y compris l'ammoniaque.

\section{CONCLUSION}

La comparaison des méthodes de recherche des neutralisants montre que :

$1^{\circ}$ Pour les laits dont l'analyse donne une teneur en acide lactique dosable en corrélation avec l'acidité titrable (ou Dornic), l'absence de neutralisant est évidente.

$2^{\circ}$ Pour les laits dont l'acidité lactique n'est pas en rapport avec l'acidité Dornic, la présence de neutralisant doit être confirmée par une ou plusieurs autres déterminations en tenant compte des variations naturelles.

IL EST DONC NÉCESSATRE DE COMMENCER PAR LE DOSAGE DES LACTATES ET DE L'ACIDE LACTIQUE. 


\title{
SUMMARY
}

Several methods based on different principles are used to analyse liquid milks. It is necessary to know the amplitude of natural variations of milk composition and to study the possible sources of error in order to appreciate the value of each procedure.

There are two methods for determining the alkalinity of ashes compared; the A. O. A. C. method gives more reproductible results than the Dutch method; but neither of them can be used for the examination of ammonia neutralized milks.

With flame photometry the determination of alkaline and alkaline-earth metals is possible. A direct method is described to titrate ammonia.

For the determination of lactates and lactic acid, Davidson, Venekamp and Kruisheer methods give comparable results; the first may be used as a standard method; the second, which is more rapid, is more convenient for mass analysis.

\section{REVUE}

\section{LE LAIT DANS LE MONDE}

\author{
par \\ G. GÉNIN \\ Ingénieur E. P. C.
}

\section{La situation de I'Industrie Laitière dans le monde}

Le rapport pour 1959, de l'Organisation des Nations Unies, pour les produits alimentaires et l'agriculture, a été publié récemment et on trouvera parmi les nombreux renseignements qu'il donne, d'utiles informations sur l'évolution de l'industrie laitière dans le monde.

Devant le développement régulier de la production laitière constatée dans certains pays, le Royaume-Uni, les Etats-Unis et différents autres pays ont réduit l'aide qu'ils apportaient aux producteurs de lait, aide destinée à maintenir les prix, afin de décourager la production laitière. En particulier, on constate une importante crise dans le marché du beurre, ce qui a obligé les gouvernéments à prendre des mesures et il est certain que ces décisions serviront de leçon pour l'avenir. 